\title{
A Comparative Study of Hand Hygiene Practices in Operation Theaters in Tertiary Level Hospitals in Delhi, India
}

\author{
${ }^{1}$ Parmeshwar Kumar, ${ }^{2}$ Shakti Kumar Gupta, ${ }^{3}$ Arti Kapil, ${ }^{4}$ Aarti Vij, ${ }^{5}$ IB Singh
}

\begin{abstract}
Background: Healthcare associated infections (HCAls) are directly related to the hand hygiene practices. Differences in implementation of practices may exist among hospitals despite standard guidelines.
\end{abstract}

Objective: To compare the hand hygiene practices in the operation theaters of tertiary care hospitals in Delhi.

Design and setting: A6-months descriptive and cross-sectional study conducted in operation theaters of tertiary level, referral public and private sector hospitals in Delhi.

Materials and methods: Six leading multispecialty hospitals, three each from the private and public sectors were selected through purposive sampling. The sample comprised of cases from one major operation theater (OT) from each hospital conducting general surgery cases ( $10 \%$ of all cases).

A performa with 24 parameters was designed using the Center for Disease Control Guidelines for hand hygiene. Hospitals were analysed in categories and also independently.

Results: One thousand nine hundred and twenty observations were analyzed from six hospitals. The level of compliance was higher among the private sector and the autonomous hospital. Statistically significant differences were observed with groups of hand hygiene parameters namely hand washing, selection of hand hygiene agent, skin care, and educational programs and surgical scrub, but not regarding hand hygiene policy or technique. Comparison of five hand hygiene practices strongly recommended by CDC practices revealed significant differences. Adherence to hand washing practices was $76 \%$,

\footnotetext{
${ }^{1}$ Assistant Professor, ${ }^{2}$ Medical Superintendent

${ }^{3-5}$ Professor

${ }^{1}$ Department of Hospital Administration, Sanjay Gandhi Postgraduate Institute of Medical Sciences, Lucknow, Uttar Pradesh, India

${ }^{2} \mathrm{Dr}$ RP Centre for Ophthalmic Sciences, All India Institute of Medical Sciences, New Delhi, India

${ }^{3}$ Department of Microbiology, All India Institute of Medical Sciences, New Delhi, India

4,5Department of Hospital Administration, All India Institute of Medical Sciences, New Delhi, India
}

Corresponding Author: Parmeshwar Kumar, Assistant Professor, Department of Hospital Administration, Sanjay Gandhi Postgraduate Institute of Medical Sciences, Raebareli Road, Lucknow-226014, Uttar Pradesh, India, Phone: 08765977620, e-mail: drpkaiims@gmail.com surgical scrub practice was $85 \%$ and overall compliance of hand hygiene practice was $80.5 \%$.

Conclusion: The study revealed gaps in implementation of hand hygiene practices despite standard guidelines. In future, post interventional studies may reflect the extent of improvement of these practices through reduction in HCAls.

Keywords: Hand hygiene practices, Operation theater, Surgical scrub.

How to cite this article: Kumar P, Gupta SK, Kapil A, Vij A, Singh IB. A Comparative Study of Hand Hygiene Practices in Operation Theaters in Tertiary Level Hospitals in Delhi, India. Int J Res Foundation Hosp Healthc Adm 2014;2(2):87-93.

Source of support: Nil

Conflict of interest: None declared

\section{INTRODUCTION}

Hand hygiene is a general term that applies to either hand washing, antiseptic hand wash, antiseptic hand rub, or surgical scrub. ${ }^{2}$

Healthcare associated infections (HCAIs) are directly related to the hand hygiene practices. The increase in HCAI would mean an increase in cost incurred by the institution and the patient. Considering the resource and financial constraints as well as higher patient load in Indian hospitals, following the standard hand hygiene practices is vital. ${ }^{1}$ Although, standard guidelines exist, there may be differences in implementation of the guidelines among hospitals especially in the absence stringent healthcare regulations. ${ }^{13}$ The study was undertaken to compare the hand hygiene practices in the operation theaters of tertiary care referral hospitals in Delhi.

\section{MATERIALS AND METHODS}

Study setting: Tertiary level hospitals in Delhi, with three hospitals each from the private and public sectors selected through purposive sampling based on permission from authorities.

Study period: The study was conducted for a period of 6 months from Oct 2010 to April 2011 commenced after obtaining clearance from ethics committee.

Study design: The study was descriptive and crosssectional in nature. 
Study sample: Comprised of one major operation theater each from the six hospitals. Only general surgery cases were selected, since it was felt that comparing hand hygiene practices of disparate specialties between different hospitals would not elicit accurate results. Specialized trauma care hospitals, single speciality hospitals and septic or emergency operation theaters were excluded from the study.

Keeping in view the constraints of time allotted by the hospitals for the study, $10 \%$ of the total number of general surgery cases being operated during the month was studied as sample of the population. Accordingly, 15 cases from autonomous hospital and 13 cases each from the other five hospitals were studied (a total of 80 cases). The auditees included all the surgical personnel associated with the cases including the scrubbed and the non-scrubbed staff.

\section{Tools}

\section{Performa}

The Center for Disease Control Guidelines for hand hygiene in healthcare settings (monthly morbidity and mortality weekly report-2002) was taken as a base document to design a performa comprising of 24 parameters. This was pilot tested and used as the tool.

The performa was divided into seven groups (Table 1).

\section{Data Processing and Analysis}

For convenience of processing the data, the six hospitals were divided into three categories:

- Public sector hospitals - A and B

- Accredited private sector hospitals - Hospitals C, D and E

- Autonomous government hospital-Hospital F

However, on occasions where the parameter itself warranted separate analysis, or were strongly recommended (category 1A) by CDC guidelines, interhospitals analysis also performed.

Statistical significance of the difference between categories/individual hospitals were determined using the fisher's exact test and chi-square test to calculate the p-values. The software SPSS Version -16.0 and software Stata 9.1 was used as the tool.

\section{RESULTS}

A total of 1920 observation were made from 80 cases studied in the six hospitals. The outcomes have broadly divided into the following heads (Table 1):

1. Parameters related to hand washing practices (parameters 1-16): groups 1 to 6

2. Parameters pertaining to surgical scrub (parameters 17-24): group 7

1. Hand washing: The overall compliance with the eight parameters was $74.6 \%$, with public sector hospitals having compliance of $66 \%$, private sector $-76 \%$ and autonomous - 87\% $(\mathrm{A}=70 \%, \mathrm{~B}=62 \%, \mathrm{C}=84 \%, \mathrm{D}=$ $86 \%, \mathrm{E}=59 \%, \mathrm{~F}=87 \%$ ) (Table 2). Results with certain individual parameters deserve mention. Decontamination of hands before individual catheter insertion, etc. that do not require special surgical procedure was only being followed $71 \%$ cases in public sector hospitals as against $95 \%$ in private sector and $100 \%$ cases in autonomous hospital. Decontamination after contact with patient's intact skin was only $50 \%$ among public sector hospitals as against $79.3 \%$ in private hospitals and $100 \%$ in autonomous institution. Adherence to practice of hand washing after removal of gloves was poor $(60 \%)$ except in the autonomous institution (100\%). The compliance with hand washing practices before use of cafeteria service was $100 \%$.

When the hospitals were compared using statistical tools, the difference in hand washing practices was statistically significant $(\mathrm{p}<0.001)$ (Table 3 ) with public sector institutions meeting all eight parameters on only six occasions (out of 26 cases), private sector on 20 (out of 39 cases) and autonomous on all 15 occasions.

2. Hand hygiene technique: Personnel in all hospitals followed the World Health Organization (WHO) recommended hand hygiene technique for hand washing (Table 2).

3. Hand hygiene agents: The hand washing agents being used were all found to be as recommended by the CDC guidelines. There were as follows:

a. Soap based anti-septic hand wash: Sodium Laureth sulphate and $\mathrm{NaCl}$ were used in public sector hospital A.

Table 1: Groups of hand hygiene parameters

\begin{tabular}{clc}
\hline Groups & Groups of hand hygiene parameters & No. of parameters \\
\hline 1. & Hand washing and hand antiseptic & 8 \\
2. & Hand hygiene technique & 1 \\
3. & Selection of hand hygiene agents & 2 \\
4. & Skin care & 1 \\
5. & Policy regarding other aspects of hand hygiene & 2 \\
6. & Healthcare worker educational and motivational programs & 2 \\
7. & Surgical scrub & 8 \\
\hline Total & & 24
\end{tabular}

Note: Groups 1-6 pertain to hand washing parameters and group 7 pertains to surgical scrub parameters 
Table 2: Hospital-wise compliance of hand washing parameters against guidelines

\begin{tabular}{|c|c|c|c|c|c|c|c|}
\hline \multirow[t]{2}{*}{ Groups } & \multicolumn{2}{|c|}{ Public sector (68.2\%) } & \multicolumn{3}{|c|}{ Private sector (75.2\%) } & \multirow{2}{*}{$\frac{\text { Autonomous (84.7\%) }}{F}$} & \multirow{2}{*}{$\begin{array}{l}\text { Overall } \\
\text { compliance }\end{array}$} \\
\hline & $A$ & $B$ & $C$ & $D$ & $E$ & & \\
\hline Hand washing & $70 \%$ & $62 \%$ & $84 \%$ & $86 \%$ & $59 \%$ & $87 \%$ & $74.6 \%$ \\
\hline Hand hygiene technique & $100 \%$ & $100 \%$ & $100 \%$ & $100 \%$ & $100 \%$ & $100 \%$ & $100 \%$ \\
\hline Hand hygiene agent & $100 \%$ & $50 \%$ & $73 \%$ & $50 \%$ & $88 \%$ & $100 \%$ & $77 \%$ \\
\hline Skin care & $0 \%$ & $0 \%$ & $0 \%$ & $100 \%$ & $0 \%$ & $80 \%$ & $30 \%$ \\
\hline Policy & $77 \%$ & $92 \%$ & $77 \%$ & $100 \%$ & $100 \%$ & $80 \%$ & $85 \%$ \\
\hline Educational programs & $84 \%$ & $77 \%$ & $100 \%$ & $100 \%$ & $85 \%$ & $50 \%$ & $82.6 \%$ \\
\hline Total & & & & & & & $75.0 \%$ \\
\hline
\end{tabular}

Table 3: Statistical significance of hand washing parameters among categories of hospitals

\begin{tabular}{llllll}
\hline Groups & $\begin{array}{l}\text { No. of } \\
\text { parameters }\end{array}$ & $\begin{array}{l}\text { Public sector } \\
(N=26) n^{\#}\end{array}$ & $\begin{array}{l}\text { Private sector } \\
(N=39) n^{\#}\end{array}$ & $\begin{array}{l}\text { Autonomous hospital } \\
(N=15) n^{\#}\end{array}$ & $\begin{array}{l}p \text {-value } \\
15(100 \%)\end{array}$ \\
\hline Hand washing & 8 & $6(23 \%)$ & $20(51.2 \%)$ & $<0.001$ & - \\
Hand hygiene technique & 1 & $26(100 \%)$ & $39(100 \%)$ & $15(100 \%)$ & $15(100 \%)$ \\
Hand hygiene agent & 2 & $26(100 \%)$ & $28(73 \%)$ & $3(20 \%)$ & $<0.001$ \\
Skin care & 1 & $0(0 \%)$ & $13(33.3 \%)$ & $10(73.3 \%)$ & 0.061 \\
Policy regarding hand hygiene & 2 & $17(69.2 \%)$ & $33(84.6 \%)$ & $9(60 \%)$ & 0.008 \\
Educational programs & 2 & $15(61.5 \%)$ & $35(89.7 \%)$ & 901 \\
\hline
\end{tabular}

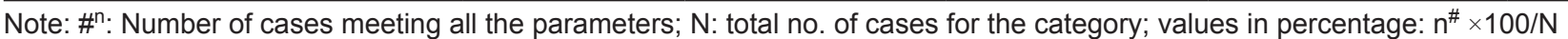

b. $0.4 \%$ chlorhexidine hand wash: The autonomous institution used this product on a majority of occasions - $43 \%$. Only one public sector hospital B used it ( $28 \%$ occasions).

c. Chlorhexidine $0.5 \%$ with ethanol combination: This product was preferred in the private sector hospitals; overall use $-33 \%$ cases.

d. Alcohol based (75\% isopropyl alcohol) hand rubs: This was used in all hospitals; most commonly before or after direct contact with patients; overall use - $33 \%$.

e. Iodine preparations (5\% betadine or $7 \%$ povidone iodine): The private sector hospitals had an overall use of $35 \%$ for iodine based products, while the other hospitals did not use it for this purpose.

f. Non-antimicrobial soap: Only hospital B (17\%), hospital E staff (on $44 \%$ the occasions) used nonantimicrobial soap for hand washing. The overall use of non-antimicrobial soap was 8 and $21 \%$ in public and private sector hospitals respectively.

All healthcare workers (HCWs) in both public sector hospitals and autonomous hospital claimed satisfaction with the products. Contrastingly, $65 \% \mathrm{HCWs}$ across the spectrum in private hospitals complained of some form of irritancy with products currently in use.

The procedure of topping-off of partially empty dispensers with soap with known propensity for bacterial contamination has been discouraged in $100 \%$ cases in all hospitals except private hospital $\mathrm{E}$ which had $0 \%$ compliance (Table 2).

4. Skin care: Eighty percent of the workers in the autonomous hospital and $100 \%$ workers in private hospital D reported to have been provided with emollients and creams.
5. Policy regarding other aspects of hand hygiene, such as wearing of gloves by non-scrubbed staff likely to come in contact with blood and other infectious material (BOIM), revealed that no HCWs in any hospitals except private hospitals B $(100 \%$ cases $)$ wore gloves.

6. Health workers' awareness and motivational programs: The autonomous hospital had only $20 \%$ of staff who had attended any hand hygiene awareness program. All other hospitals had personnel having attended some form of education program. In $80 \%$ of cases in hospital $\mathrm{F}$, there were systems in place for monitoring adherence to recommended hand hygiene practices. The public sector hospitals had monitoring only in $61.5 \%$ cases.

The differences between the categories of hospitals were statistically significant with respect to the groups of hand washing, selection of hand hygiene agents and skin care (Table 3).

Strongly recommended hand hygiene practices: Five hand hygiene practices have been considered essential and are strongly recommended under the category $1 \mathrm{~A}$ of $\mathrm{CDC}$ guidelines.

Interhospital comparison between the groups of hospitals regarding the strongly recommended hand hygiene practices is shown in Graph 1.

The autonomous hospital had an overall compliance of $80 \%$ followed by private hospitals with $67.8 \%$ and then by public sector hospital with $62 \%$ compliance with the strongly recommended practices.

This was despite the observation that $28 \%$ of cases in private sector met with all five of the parameters as against the autonomous which had only $6.6 \%$ cases meeting all five practices. On no occasion did the public sector hospital meet with all the five strongly recommended practices. 
The differences were statistically significant $(\mathrm{p}<0.001)$.

The overall compliance with hand washing practices was $75 \%$ (Graph 2); the level of compliance with standards higher among the private sector and the autonomous hospital.

\section{Surgical Scrub}

Compliance for surgical scrub practices against the guidelines was $85 \%$. The category wise compliance was as follows (Graph 3):

- Autonomous hospital F-91\%

- Public sector-83\%

a. Hospital A-70\%

b. Hospital B- $80 \%$

- Private sector $-80 \%$

a. Hospital C-67\%

b. Hospital D- $82 \%$

c. Hospital E- $80 \%$

The comparison as three categories was not significant $(\mathrm{p}=0.09)$ but as individual hospitals, the difference was statistically significant $(\mathrm{p}<0.001)$.

All HCWs observed in public sector hospitals and autonomous hospital $\mathrm{F}$ adhered to the recommended practice of removal of rings, watches, etc. while scrubbing as against $74 \%$ in private hospitals.

Only $47 \%$ of scrubbing staff in hospital F and $92 \%$ of hospital E performed the recommended cleaning of subungual region before the first case of the day. All others practiced it on $100 \%$ occasions. Healthcare worker in all hospitals except hospital F (80\%) kept their fingernails shortened.
The technique followed for alcohol based or antiseptic soap based scrub was correct in all cases. However, only personnel in hospital $\mathrm{F}$ gave sufficient time (4-8 minutes) on all occasions for hands to dry before donning gloves.

The overall compliance among hospitals for hand hygiene practices against standard guidelines was $80.5 \%$. Category-wise autonomous hospital $-89 \%$, private sector $-78 \%$, public sector $-74.5 \%$ (Graph 4$)$.

\section{DISCUSSION}

The study reveals a significant gap between hand hygiene practices in operation theaters in Delhi and that promulgated by standard guidelines, and more so in case of hand washing than surgical scrubs. The autonomous hospital performed better than private and public sector hospitals when analysed as categories and individually, on several parameters. Adherence to hand washing practices with non-surgical procedures, such as catheter insertion and decontamination after contact with intact skin and removal of gloves was poorer in public sector hospitals. This is in keeping with the general propensity of lower compliance with nonsurgical procedures. In a study in 2013 of 207 procedures of catheter insertion revealed $57.9 \%$ of hand disinfection before patient contact and $45.5 \%$ hand disinfection before aseptic procedure.

Since, the publication of the WHO guidelines in $2009,{ }^{3}$ there has been a global awareness about hand hygiene techniques could probably explain the correct practice of hand hygiene techniques by the operating rooms personnel across all hospitals. Though, all hospitals used CDC

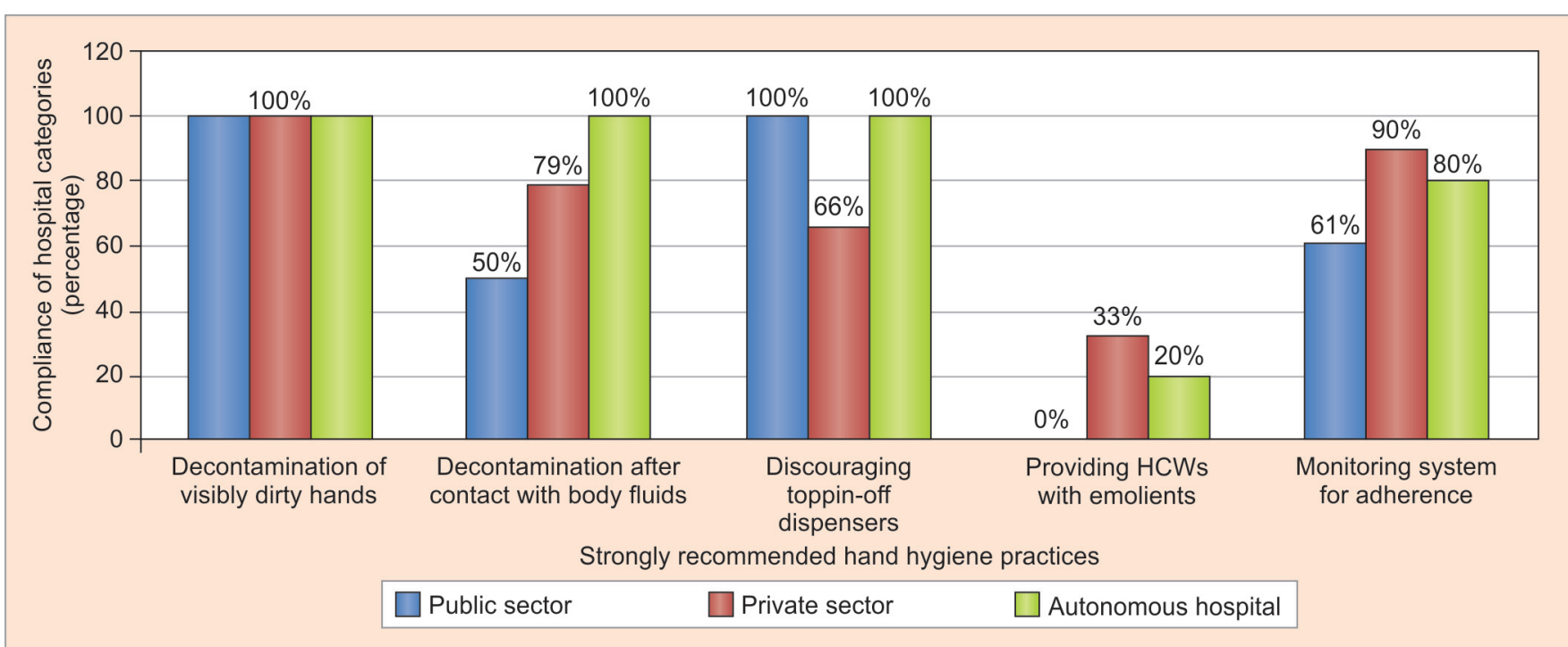

Graph 1: Comparison among categories of hospitals for strongly recommended hand hygiene practices. The practices are hand washing with soap and water in case of hands visibly dirty or soiled with BOIM, decontamination with antimicrobial soap and water or alcohol-based hand rub after contact with body fluids excretions, mucous membrane, etc. if hands are not visibly soiled, topping-off of half empty soap dispensers with soap is to be discouraged, hand lotions or creams are recommended to be provided to the HCWs to minimize the occurrence of irritant contact dermatitis and a monitoring system must be in place to ensure adherence of HCWs to the hand hygiene practices 


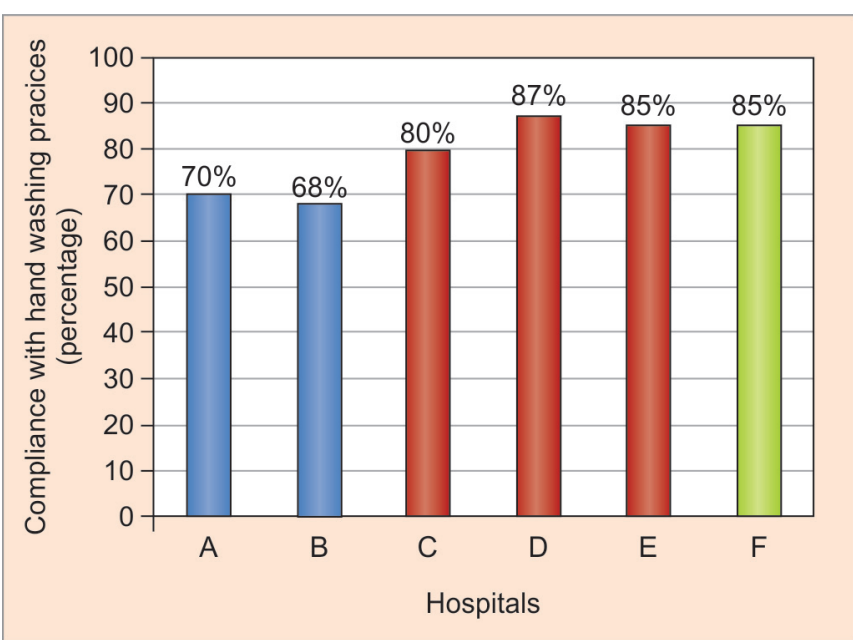

Graph 2: Compliance of hand washing practices among individual hospitals. A and B represent public sector hospitals; C, D and E are private sector hospitals and $\mathrm{F}$ is autonomous hospital

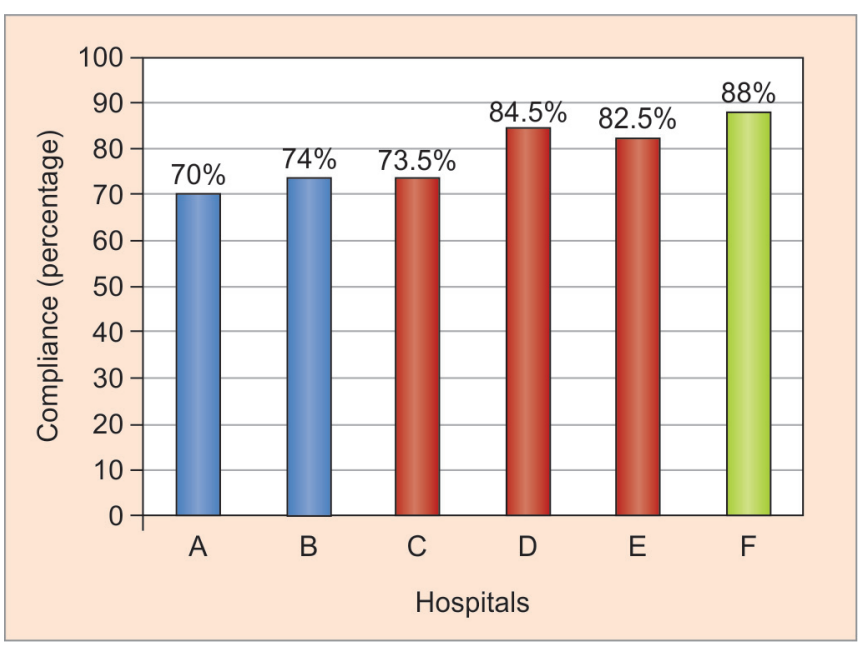

Graph 4: Overall compliance of hand hygiene practices among hospitals. These include hand washing practices and surgical scrub practices. A and B represent public sector hospitals; C, D and E are private sector hospitals and $\mathrm{F}$ is autonomous hospital

recommended hand hygiene agents, the choice of product differed significantly. Hundred percent of the HCWs in both public sector hospitals and autonomous hospital claimed satisfaction with the products Iodine based products, known to have some irritancy potential, ${ }^{6}$ were used exclusively by private sector hospitals and may account for the finding that $65 \% \mathrm{HCWs}$ in private hospitals complained of some form of irritancy with products. This is despite the fact all HCWs in private hospitals and no HCW in public sector hospitals were provided with emollients, known to reduce contact dermatitis. ${ }^{11}$ Unlike studies in the western world, the adherence with hand washing practices before use of cafeteria service was very high. This may reflect not only difference in eating habits deep rooted cultural or even religious beliefs related to hand washing, but also the concept of inherent hand hygiene, a subject in the realm of behavioral sciences. ${ }^{8}$

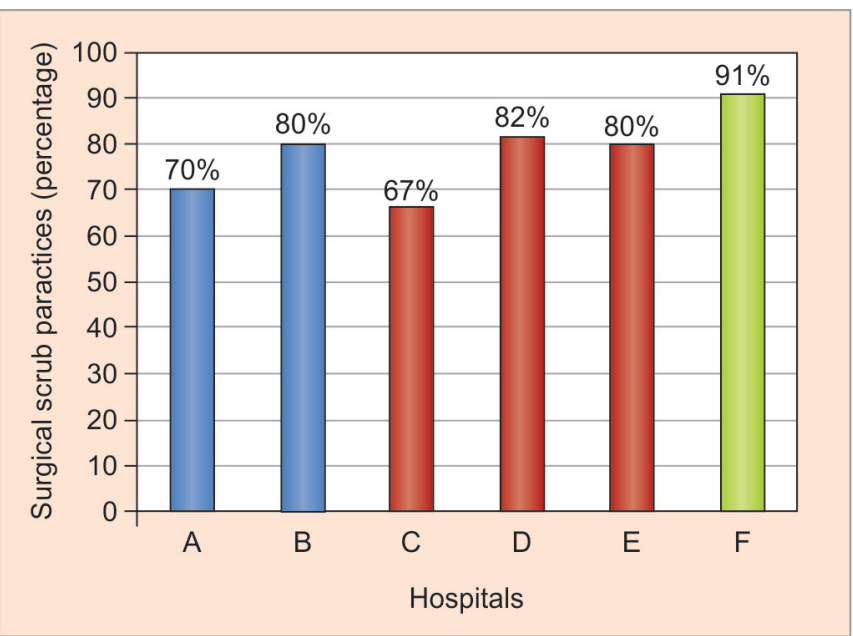

Graph 3: Interhospital comparison of surgical scrub practices. $A$ and $B$ represent public sector hospitals; C, D and $E$ are private sector hospitals and $\mathrm{F}$ is autonomous hospital

Five hand hygiene practices considered essential and strongly recommended under the category $1 \mathrm{~A}$ of $\mathrm{CDC}$ guidelines are:

- Hand washing with soap and water is recommended in case of hands visibly dirty or soiled with BOIM.

- Hand decontamination is essential with antimicrobial soap and water or alcohol based hand rub after contact with body fluids excretions, mucous membrane, etc. if hands are not visibly soiled.

- Topping-off of half empty soap dispensers with soap is to be discouraged.

- Hand lotions or creams are recommended to be provided to the HCWs to minimize the occurrence of irritant contact dermatitis.

- A monitoring system must be in place to ensure adherence of HCWs to the hand hygiene practices.

An analysis of individual hospital reveals that the order of compliance with strongly recommended practices is - Hospital D (private) $>\mathrm{F}$ (autonomous) $>\mathrm{C}$ (private) $>\mathrm{A}$ (public) $>\mathrm{E}$ (private) $>\mathrm{B}$ (public), the difference is statistically significant. The practice of topping-off of partially empty dispensers with soap with known propensity for bacterial contamination was observed in private hospitals and probably reflects an attempt at cost containment. Although, only a small percentage of staff at the autonomous hospital had attended any hand hygiene awareness program, majority of the staff in that hospital ( $80 \%$ ) claimed that there were systems in place for monitoring adherence to recommended hand hygiene practices. The role of educational programs in better compliance in $\mathrm{HH}$ practices has been studied extensively and endorsed. ${ }^{4}$

Compliance for surgical scrub practices against the guidelines was high (85\%). Relatively lower adherence to practices, such as removal of rings prior to surgery by 
private hospital staff, indicate a lack of policy implementation, despite several study findings demonstrated that skin underneath rings is more heavily colonized than comparable areas of skin on fingers without rings. ${ }^{13,5}$ Despite scoring high in most scrub related parameters, autonomous hospitals did not appear to have a policy on subungual practices. Though, CDC recommends that the first scrub of the day must include cleaning underneath fingernails and studies prove that natural nails tips should be kept short to prevent SSI, the autonomous hospital fared far worse than other hospitals in this regard.

In keeping with global trends, Povidone-iodine and chlorhexidine gluconate were observed to be agents of choice among than surgical team members. Both are recommended by the CDC guidelines despite chlorhexidine having longer residual effect, since both have been proven to be equally efficacious in reducing chances of surgical site infection (SSI). ${ }^{9}$ Surprisingly, despite the higher patient load in autonomous hospital, only HCWs in this hospitals allowed sufficient time for drying of $\mathrm{HH}$ agent before donning gloves.

As an endorsement of training and good policy implementation all scrubbed staff was observed to have followed the correct technique of surgical scrub again a result of the WHO focus on safe surgery. ${ }^{14}$

A limitation of the study was that, although covert observation was attempted, the findings may not precisely reflect actual practices as a result of Hawthorne effect. Additionally, a larger sample size of hospitals may have helped in offsetting the effect of poorly performing hospital in a particular group.

Despite the drawbacks, the study illustrates that adherence to hand-hygiene guidelines by OT staff is low, which potentially increases the risk of HCAIs. Significant gap was observed in hand hygiene practices between hospitals and against the standard guidelines. Importantly, the study helped to identify those parameters which were exclusively being non-complaint by specific category of hospitals and some deficiencies that may serve as representative all categories of hospitals in India.

Adherence to $\mathrm{HH}$ practices requires a multi-factorial approach. In a landmark study, the investigators identified hospital wide predictors of poor adherence to recommended hand hygiene measures. ${ }^{10}$ These included professional category, time of day/week, type and intensity of patient care. Future studies need to focus on these predictors especially in reference to operation theaters. Several studies have elucidated educational and behavioral interventions for better adherence. ${ }^{3,7}$ They have also revealed perceived barriers to adherence, such as skin irritation caused by hand hygiene agents, inaccessible hand hygiene supplies, interference with $\mathrm{HCW}$ - patient relationships, patient needs perceived as apriority over hand hygiene, wearing of gloves, forgetfulness, lack of knowledge of guidelines, insufficient time for hand hygiene and high workload and understaffing. Similar studies in Indian settings are essential to identify the gaps and reduce HCAI. Post interventional studies will assist in not only channelizing limited resources toward achieving the best outcome but also help in customizing the intervention measures to the groups of healthcare personnel.

This may pave the way for the formulation of a standardized hand hygiene policy for operation theaters across the country thereby ensuring a better healthcare delivery system. As a cornerstone of patient safety, hand hygiene practices would also go a long way in making the hospitals a safer place, both for the patients and the HCWs.

\section{ACKNOWLEDGMENTS}

We thank the administration of all the hospitals for granting permission to conduct the study in their hospital. We also wish to acknowledge the support lent by the surgical staff in the operation theaters.

\section{REFERENCES}

1. Camins BC, Fraser VJ. Reducing the risk of healthcare associated infections by complying with $\mathrm{CDC}$ hand hygiene guidelines. Joint Commission J Quality and Patient Safety 2005 Mar;31(3):173-179.

2. Centers for Disease Control. Guidelines for Hand hygiene in healthcare settings. MMWR 2002;51 (No. RR-16):3.

3. Conly JM, et al. Hand washing practices in an intensive care unit: the effects of an educational program and its relationship to infection rates. Am J Infect Control 1989;17:330-339.

4. Doron SI, Kifuji K, Hynes BT, Dunlop D, et al. A multifaceted approach to education, observation, and feedback in a successful hand hygiene campaign. Jt Comm J Qual Patient Saf 2011 Jan;37(1):3-10.

5. Field EA, McGowan P, Pearce PK, Martin MV. Rings and watches: should they be removed prior to operative dental procedures? J Dent 1996 Jan-Mar;24(1-2):65-69.

6. Kampf G1, Reise G, James C, Gittelbauer K, Gosch J, Alpers B. Improving patient safety during insertion of peripheral venous catheters: an observational intervention study. GMS Hyg Infect Control 2013 Nov 6;8(2):18.

7. Kretzer EK, Larson EL. Behavioural interventions to improve infection control practices. Am J Infec Control 1998 Jun;26(3): 245-253.

8. O'Boyle CA, Henly SJ, Larson E. Understanding adherence to hand hygiene recommendations: the theory of planned behavior. Am J Infect Control 2001 Dec;29(6):352-360.

9. Jarral OA, McCormack DJ, Ibrahim S, Shipolini AR. Should surgeons scrub with chlorhexidine or iodine prior to surgery? Interact Cardiovasc Thorac Surg 2011 Jun;12(6):1017-1021.

10. Pittet D, Mourouga P, Perneger TV. Compliance with hand washing in a teaching hospital. Ann Int Medi 1999 Jan 19; 130(2):126-130. 
11. Visscher M. Effect of topical treatments on irritant hand dermatitis in healthcare workers. Am J Infect Control 2009 Dec 1;37(10):842.

12. WHO guidelines on hand hygiene in healthcare. First global patient safety challenge clean care is safer care. Geneva: World Health Organization; 2009. Available at: http:// whqlibdoc.who. int/publi-cations/2009/9789241597906_eng.pdf. Web accessed on 12.06.13.
13. Wynd CA, Samstag DE, Lapp AM. Bacterial carriage on the fingernail of OR nurses. AORN J 1994 Nov;60(5):796, 799-805.

14. WHO guidelines on safe surgery 2009. Second global patient safety challenge safe surgery saves lives. Geneva: World Health Organization; 2009. Available at: http://whqlibdoc.who.int/ publications/2009/9789241598552_eng.pdf. Web accessed on 02.3.11. 